\title{
Strong Limit Theorems for Dependent Random Variables
}

\author{
Libin Wu, Bainian Li \\ School of Stat \& Applied mathematics Anhui University of \\ Finance and Economics, Bengbu 233030, PR China
}

Received: February 17, 2021. Revised: March 9, 2021. Accepted: March 12, 2021. Published: March 26, 2021.

\begin{abstract}
In this article We establish moment inequality of dependent random variables, furthermore some theorems of strong law of large numbers and complete convergence for sequences of dependent random variables. In particular, independent and identically distributed Marcinkiewicz Law of large numbers are generalized to the case of mo -dependent sequences..
\end{abstract}

Keywords-Lacunary System, Generalized Gaussian, NA sequences, strong law of large numbers

\section{INTRODUCTION}

$\mathrm{L}$ et $X_{1}, X_{2}, \cdots$, denote a sequence of random varibles $\Lambda_{\text {defined on a fixed probability space }(\Omega, F, P)}$.The partial sums of the random variables are

$$
\begin{aligned}
& S_{n}=\sum_{i=1}^{n} X_{i}, \text { for } n \geq 1 \text { and } S_{0}=0, \\
& S_{b, n}=\sum_{i=b+1}^{b+n} X_{i}, S(i, j)=\sum_{k=i}^{j} X_{k}, 1 \leq i \leq j .
\end{aligned}
$$

Definition 1.1 (cf. Fazekas and Klesov, 2000, p. 447)[1]. A sequence of random variables $\left\{X_{n}, n \geq 1\right\}$ is said to have the $r$ th $(r>0)$ moment function of superadditive structure if there exists a non-negative function $g(i, j)$ of two arguments such that for all $b \geq 0$ and $1 \leq k \leq k+l$

$$
\begin{aligned}
g(b, k) & +g(b+k, l) \leq g(b, k+l) \\
E\left|S_{b, n}\right|^{r} & \leq g^{\alpha}(b, n), n \geq 1, \text { for some } \alpha>1 .
\end{aligned}
$$

Definition 1.2. Let $X$ be a real-valued random variable, we call a Locally Generalized Gaussian, If there exists $\alpha>0$, such that

$$
E(\exp (u X) \mid F) \leq \exp \left(u^{2} \alpha^{2} / 2\right) \quad \text { a.s. }
$$

for any $u \in R$.

Definition 1.3. Given $p>0$, a sequence of real-valued random variables $\left\{X_{n}, n \geq 1\right\}$ is called a Lacunary System or an $S_{p}$ system, if there exists a positive constant $K_{p}$ such that

$$
E\left|\sum_{i=m}^{n} C_{i} X_{i}\right|^{p} \leq K_{p}\left(\sum_{i=m}^{n} C_{i}^{2}\right)^{p / 2}
$$

for any sequence of real constant $C_{i}$ and all $\mathrm{n} \geq \mathrm{m}$.

Definition 1.4 The random variables $X_{1}, X_{2}, \cdots, X_{n}$ are said to be negatively associated if for every $\mathrm{n}$ and every pair of disjoint subsets $A_{1}, A_{2}$ of $\{1,2, \ldots, n\}$

$$
\operatorname{Cov}\left(f_{1}\left(X_{i}, i \in A_{1}\right), f_{2}\left(X_{j}, j \in A_{2}\right)\right) \leq 0,
$$

Whenever $f_{1}$ and $f_{2}$ are coordinatewise increasing and this covariance exists.
Definition 1.5 A stationary sequence $\left\{X_{n}\right\}$ is called $m_{0}$ -dependent for a given fixed $m_{0}$ if $\left(X_{1}, X_{2}, \cdots, X_{i}\right)$ and $\left(X_{j}, X_{j+1}, \cdots,\right)$ are independent whenever $j-i>m_{0}$.

Lemma 1.1 (Dini Theorem, Fikhtengolts, 1954, p. 286)[2]. Let $c_{1}, c_{2}, \cdots$ be non-negative numbers, $v_{n}=\sum_{k=n}^{\infty} c_{k}$, if $0<v_{n}<\infty$ for $n \geq 1$, then

$$
\sum_{n=1}^{\infty} \frac{c_{k}}{v_{n}^{\delta}}<\infty, 0<\delta<1 .
$$

Lemma 1.2 (cf. Hu, 2005, Theorem 2.1.)[3] We assume that $\left\{X_{n}, n \geq 1\right\}$ has the $r$ th moment function of superadditive structure, $g(0, n)=g_{n}$ and $g_{n}$ non-decreases, $\left\{b_{n}\right\}$ is a non-decreasing unbounded sequence of positive numbers and $\sum_{n=1}^{\infty} \frac{g_{n}^{\alpha}-g_{n-1}^{\alpha}}{b_{n}^{r}}<\infty$, then

$$
\lim _{n \rightarrow \infty} \frac{S_{n}}{b_{n}}=0 \quad \text { a.s. }
$$

and with the growth rate

$$
\frac{S_{n}}{b_{n}}=O\left(\frac{\beta_{n}}{b_{n}}\right) \quad \text { a.s., }
$$

where $\beta_{n}=\max _{1 \leq k \leq n} b_{k} v_{k}^{\delta / r}, 0<\delta<1, v_{n}=n \sum_{k=1}^{\infty} \frac{\alpha_{k}}{b_{k}^{r}}, \lim _{n \rightarrow \infty} \frac{\beta_{n}}{b_{n}}=0$.

Lemma $1.3\left(\mathrm{Hu}, 2005\right.$, Lemma1.2)[3] Let $b_{1}, b_{2}, \cdots$ be a non-decreasing unbounded sequence of positive numbers and $a_{1}, a_{2}, \cdots$ be nonnegative numbers. Let $r$ and $C$ be fixed positive numbers. Assume that for each $n \geq 1$

$$
\begin{gathered}
E\left(\max _{1 \leq l \leq n}\left|S_{l}\right|\right)^{r} \leq C \sum_{l=1}^{n} \alpha_{l}, \\
\sum_{l=1}^{n} \frac{\alpha_{l}}{b_{l}^{r}}<\infty .
\end{gathered}
$$

then (5) and (6) hold .

Lemma 1.4 (Yang, 2000, Corollary 3, Yang, 2001; Shao, 2000)[4][6][7]. Let $X_{1}, X_{2}, \cdots$ be negatively associated random variables with zero means and $E\left|X_{l}\right|^{r}<\infty$, where $r>1$. Then there exists a positive constant $C$, which does not depend on $n$, such that

$$
\begin{aligned}
& E\left(\max _{1 \leq k \leq n}\left|S_{k}\right|^{r}\right) \leq C \sum_{j=1}^{n} E\left|X_{j}\right|^{r}, \quad 1<r \leq 2 \\
& E\left(\max _{1 \leq k \leq n}\left|S_{k}\right|^{r}\right) \leq C\left[\sum_{j=1}^{n} E\left|X_{j}\right|^{r}+\left(\sum_{j=1}^{n} E X_{j}^{2}\right)^{r / 2}, r>2\right.
\end{aligned}
$$


In this paper, we assume that $C, C_{1}, \cdots$ are some positive constants (not necessarily always the same) independent of $n$.

\section{MAIN RESUltS}

Theorem 2.1 Assume that $\left\{X_{n}, n \geq 1\right\}$ be a Lacunary System, exists a positive constant $K_{p}$ and $p>2$, such that $\sup E\left|X_{j}\right|^{p}<\infty$, then for every $\delta>0$,

$$
\lim _{n \rightarrow \infty} \frac{S_{n}}{\sqrt{n}(\log n)^{1 / p}(\log \log n)^{(1+\delta) / p}}=0 \quad \text { a.s., }
$$

and for $b_{n}=\sqrt{n}(\log n)^{1 / p}(\log \log n)^{(1+\delta) / p}, n \geq n_{0}$,

$$
\begin{gathered}
\alpha_{n}=K_{p} n^{p / 2}-K_{p}(n-1)^{p / 2}, \beta_{n}=\max _{1 \leq k \leq n} b_{k} v_{k}^{\delta / p}, 0<\delta<1, \\
\frac{S_{n}}{b_{n}}=O\left(\frac{\beta_{n}}{b_{n}}\right) \quad \text { a.s., } \lim _{n \rightarrow \infty} \frac{\beta_{n}}{b_{n}}=0 .
\end{gathered}
$$

Proof From definition 1.3 , for any sequence of real constant $\left\{C_{i}\right\}$

$$
E\left|\sum_{i=b+1}^{b+n} C_{i} X_{i}\right|^{p} \leq K_{p}\left(\sum_{i=b+1}^{b+n} C_{i}^{2}\right)^{p / 2},
$$

in particularly where $C_{i} \equiv 1$, we have

$$
E\left|\sum_{i=b+1}^{b+n} C_{i} X_{i}\right|^{p}=E\left|S_{b, n}\right|^{p} \leq K_{p} n^{p / 2},
$$

In Definition 1.1 take $g_{n}=g(b, n)=K_{p}^{2 / p} n, \alpha=\frac{p}{2}$, then

$$
\begin{aligned}
& g(b, k)=K_{p}^{2 / p} k, g(b+k, l)=K_{p}^{2 / p} l, \\
& g(b, k)+g(b+k, l)=K_{p}^{2 / p}(k+l) \leq g(b, k+l), \\
& E\left|S_{b, n}\right|^{p} \leq K_{p} n^{p / 2}=g^{p / 2}(b, n), n \geq 1, p>2,
\end{aligned}
$$

we know that $\left\{X_{n}, n \geq 1\right\}$ has the pth moment function of superadditive structure, and

$$
\begin{aligned}
\sum_{n=n_{0}}^{\infty} \frac{g_{n}^{p / 2}-g_{n-1}^{p / 2}}{b_{n}^{p}} & =\sum_{n=n_{0}}^{\infty} \frac{K_{p}\left(n^{p / 2}-(n-1)^{p / 2}\right)}{n^{p / 2}(\log n)(\log \log n)^{1+\delta}} \\
& \leq C \sum_{n=n_{0}}^{\infty} \frac{1}{n(\log n)(\log \log n)^{1+\delta}}<\infty
\end{aligned}
$$

thus (9) follows from Lemma1.2.

We assume that $\alpha_{n}>0$ for infinitely many n. By (8) and Lemma 1.3, we know that $\sum_{n=1}^{\infty} \frac{\alpha_{n}}{b_{n}^{r} v_{n}^{\delta}}<\infty$, it is easy to see that $0<\beta_{k} \leq \beta_{k+1}$ for $\mathrm{k} \geq 1$, and

$$
\begin{aligned}
& \sum_{n=1}^{\infty} \frac{\alpha_{n}}{\beta_{n}^{r}} \leq \sum_{n=1}^{\infty} \frac{\alpha_{n}}{\beta_{n}^{r} v_{n}^{\delta}}<\infty, \\
& \frac{\beta_{k}}{b_{k}} \leq \frac{\max _{1 \leq l \leq k_{1}} b_{l} v_{l}^{\delta / r}}{b_{k}}+\frac{\max _{k_{1} \leq l \leq k} b_{l} v_{l}^{\delta / r}}{b_{k}} \leq \frac{\max _{1 \leq l \leq k_{1}} b_{l} v_{l}^{\delta / r}}{b_{k}}+v_{k_{1}}^{\delta / r},
\end{aligned}
$$

from (8) and $\lim _{n \rightarrow \infty} b_{n}=0$, we get $\lim _{n \rightarrow \infty} \frac{\beta_{n}}{b_{n}}=0$, Eq. (7) and

Theorem 1.1 of Fazekas and Klesov (2000) imply that

$$
E\left(\max _{1 \leq l \leq n}\left|\frac{S_{l}}{\beta_{l}}\right|\right)^{r} \leq 4 C \sum_{l=1}^{n} \frac{\alpha_{l}}{\beta_{l}^{r}} \leq 4 C \sum_{l=1}^{\infty} \frac{\alpha_{l}}{\beta_{l}^{r}}<\infty,
$$

hence by monotone convergence theorem, we have

$$
\begin{aligned}
& \quad E\left(\sup _{n \geq 1}\left|\frac{S_{n}}{\beta_{n}}\right|\right)^{r}=\lim _{n \rightarrow \infty} E\left(\max _{1 \leq l \leq n}\left|\frac{S_{l}}{\beta_{l}}\right|\right)^{r} \leq 4 C \sum_{n=1}^{\infty} \frac{\alpha_{n}}{\beta_{n}^{r}}<\infty, \\
& \text { so that } \sup _{n \geq 1}\left|\frac{S_{n}}{\beta_{n}}\right|<\infty \text {, a.s., and } \\
& \qquad 0 \leq\left|\frac{S_{n}}{\beta_{n}}\right| \leq \frac{\beta_{n}}{b_{n}} \sup _{n \geq 1}\left|\frac{S_{n}}{\beta_{n}}\right|=O\left(\frac{\beta_{n}}{b_{n}}\right), \quad \text { a.s., }
\end{aligned}
$$

this completes the proof.

Remark 1 Theorem 2.1 improve result of Ryozo, Y.( Corollary 2) [8], and from strictly stationary strong mixing sequence to $S_{p}$ system.

Theorem 2.2 Let $\left(X_{n}, F_{n}\right)$ be a Locally Generalized Gaussian sequence, if $\sup X_{n}=k<\infty$, then for any $r \geq 2$

$$
\sum_{i=a+1}^{a+n} E\left|C_{i} X_{i}\right|^{r} \leq K_{r}\left(\sum_{i=a+1}^{a+n} C_{i}^{2}\right)^{r / 2}
$$

Furthermore (9) and (10) hold.

Proof Let $A_{n}=\sum_{i=a+1}^{a+n} C_{i}^{2}, u=x / k^{2} A_{n}$, by lemma 1 in [9], then

$$
E\left(\exp \left(u \sum_{i=a+1}^{a+n} C_{i} X_{i}\right)\right)=E\left(\exp \left(u S_{n}\right)\right) \leq \exp \left(u^{2} k^{2} A_{n} / 2\right),
$$

where $S_{n}=\sum_{i=a+1}^{a+n} C_{i} X_{i}$, for $r \geq 2$, by Chebyshev's inequality, we get

$$
\begin{aligned}
E & \left|\sum_{i=a+1}^{a+n} C_{i} X_{i}\right|^{r}=r \int_{0}^{\infty} x^{r-1} P\left(\left|S_{n}\right|>x\right) d x \\
& \leq 2 r \int_{0}^{\infty} x^{r-1} \exp \left(-x^{2} / 2 k^{2} A_{n}\right) d x \\
& =2^{r / 2} r k^{r} A_{n}^{r / 2} \int_{0}^{\infty} x^{r / 2-1} \exp (-x) d x \\
& =K_{r}\left(\sum_{i=a+1}^{a+n} C_{i}^{2}\right)^{r / 2}
\end{aligned}
$$

where $K_{r}=2^{r / 2} r k^{r} \int_{0}^{\infty} x^{r / 2-1} \exp (-x) d x$.

Therefore, Locally Generalized Gaussian sequence is a Lacunary system, by Theorem 2.1, (9) and (10) hold.

Theorem 2.3 Let $\left\{X_{n}, n \geq 1\right\}$ be a NA sequence, satisfying $\sup _{n}\left|X_{n}\right|^{p}<\infty$, then for any $0<p<2, \alpha p \geq 1, p / 2 \leq \delta \leq 1$, $x>0$,

$$
\sum_{n=1}^{\infty} n^{\alpha p-(1+\delta)} P\left\{\left|S_{n}\right| \geq x n^{\alpha}\right\}<\infty,
$$

where $S_{n}=\sum_{i=1}^{n} X_{i}$.

Proof From lemma 1.3, when $C_{i} \equiv 1$, we have

$$
E\left|\sum_{i=a+1}^{a+n} X_{i}\right|^{p} \leq K_{p} n^{p / 2},
$$

By Markov's inequality we get

$$
\begin{aligned}
& \sum_{n=1}^{\infty} n^{\alpha p-(1+\delta)} P\left\{\left|S_{n}\right| \geq x n^{\alpha}\right\} \leq \sum_{n=1}^{\infty} \frac{n^{\alpha p-(1+\delta)} E\left|S_{n}\right|^{p}}{x^{p} n^{\alpha p}} \\
& \leq K_{p} x^{-p} \sum_{n=1}^{\infty} \frac{n^{\alpha p-(1+\delta)} n^{p / 2}}{x^{p} n^{\alpha p}} \\
& \leq K_{p} x^{-p} \sum_{n=1}^{\infty} \frac{1}{n^{1+(\delta-p / 2)}}<\infty .
\end{aligned}
$$

Theorem 2.4 Let $\left\{X_{n}, n \geq 1\right\}$ be a $m_{0}$ dependent sequence 
with zero mean, if $\sup \left|X_{n}\right|^{r}<\infty$, then for any $1 \leq r<2$,

$$
\frac{S_{n}}{n^{1 / r}} \rightarrow 0, \quad \text { a.s. }
$$

where $S_{n}=\sum_{i=1}^{n} X_{i}$.

Proof for every $t=0,1,2, \cdots, m_{0}-1,\left\{X_{t+m+m_{0} k}, k=1,2, \cdots\right\}$ be a independent sequence, and

$$
\sum_{j=m}^{n} C_{j} X_{j}=\sum_{t=0}^{m_{0}-1} \sum_{k=0}^{n_{0}} C_{t+m+m_{0} k} X_{t+m+m_{0} k},
$$

by $C_{r}$ inequality, we get

$$
\begin{aligned}
& E\left(\left|\sum_{j=m}^{n} C_{j} X_{j}\right|^{2} \leq m_{0} \sum_{t=0}^{m_{0}-1} E\left(\sum_{k=0}^{n_{0}} C_{t+m+m_{0} k} X_{t+m+m_{0} k}\right)^{2}\right. \\
& =m_{0} \sum_{t=0}^{m_{0}-1} \sum_{k=0}^{n_{0}} C_{t+m+m_{0} k}^{2} E\left(X_{t+m+m_{0} k}\right)^{2} \\
& =m_{0} n \sum_{j=m}^{n} C_{j}^{2} E\left(X_{j}\right)^{2} \\
& \leq k m_{0} \sum_{j=m}^{n} C_{j}^{2},
\end{aligned}
$$

$\left\{X_{n}, n \geq 1\right\}$ be a $S_{2}$ system, by Jensen's inequality, for $1 \leq r<2$,

$$
E\left(\left|\sum_{j=m}^{n} C_{j} X_{j}\right|\right)^{r} \leq C\left(\sum_{j=m}^{n} C_{j}^{2}\right)^{r / 2},
$$

in particular,when $C_{i} \equiv 1$, we obtaned $E\left|S_{n}\right|^{r} \leq C n^{r / 2}$.

In Definition 1.1 take

$$
g_{n}=g(b, n)=K^{2 / r} n, \alpha=\frac{r}{2}, b_{n}=n^{1 / r}
$$

then

$$
\begin{aligned}
& g(b, k)=K^{2 / r} k, g(b+k, l)=K^{2 / r} l, \\
& g(b, k)+g(b+k, l)=K^{2 / r}(k+l) \leq g(b, k+l), \\
& E\left|S_{b, n}\right|^{r} \leq K n^{r / 2}=g^{r / 2}(b, n), n \geq 1,1 \leq r<2,
\end{aligned}
$$

we know that $\left\{X_{n}, n \geq 1\right\}$ has the $p$ th moment function of superadditive structure, and

$$
\begin{aligned}
& \sum_{n=1}^{\infty} \frac{g_{n}^{r / 2}-g_{n-1}^{r / 2}}{b_{n}^{r}}=\sum_{n=1}^{\infty} \frac{K\left(n^{r / 2}-(n-1)^{r / 2}\right)}{n} \\
& \leq C \sum_{n=1}^{\infty} \frac{n^{r / 2-1}}{n}=C \sum_{n=1}^{\infty} \frac{1}{n^{2-r / 2}}<\infty,
\end{aligned}
$$

thus (13) follows from Lemma1.2.

Remark 2 This result extends independent and identically distributed Marcinkiewicz Law of large numbers for $m_{0}$ -dependent sequences.

\section{ACKNOWLEDGMENT}

This work was supported by the Project Ministry of Education of PR China (10YJC630143) and the science Foundation of the Anhui Province (KJ2013Z001, 2011sk162).

\section{REFERENCES}

[1] Fazekas, I., Klesov, O., 2000. A general approach to the strong law of large numbers. Theory Probab. Appl. 45, 436--449..

[2] Fikhtengolts, G.M., 1954. A Course of Differential and Integral Calculus. People's Education Press, Beijing (in Chinese).

[3] Hu Shuhe,2005. A general approach rate to the strong law of large numbers.Statistics \& Probability Letters 76,843--851.

[4] Shao, Q., 2000. A comparison theorem on maximal inequalities between negatively associated and independent random variables.J. Theoret. Probab. 13 (2), 343--356..
[5] Shao, Q., Yu, H., 1996. Weak convergence for weighed empirical processes of dependent sequences. Ann. Probab. 24, 2098--2127

[6] Yang, S., 2000. Moment inequalities for partial sums of random variables. Sci. China (series A, Chinese) 30 (3), 218--223..

[7] Yang, S., 2001. Moment inequalities for partial sums of random variables. Sci. China (series A, English) 44, 1--6..

[8] Ryozo, Y., 1980. Moment Bounds for Stationary Mixing Sequences,Z. W. verw. Gebiete 52, 45-57.

LiBin Wu (1962 -), male, associate professor, master tutor, In 1984, graduated from the department of anhui normal university, bachelor. Research direction: random variable limit theory.

Bainian $\mathrm{Li}$ is Professor in the Department of information and science computer of School of Stat \& Applied mathematics Anhui University of Finance and Economics, China. His research area is strong limit theorem for dependent random variables.

\section{Creative Commons Attribution License 4.0 (Attribution 4.0 International, CC BY 4.0)}

This article is published under the terms of the Creative Commons Attribution License 4.0 https://creativecommons.org/licenses/by/4.0/deed.en US 